\title{
L'«Encyclopédie méthodique» (1782-1832). Des Lumières au Positivisme, textes publiés par Claude Blanckaert et Michel Porret
}

\section{Stefania Carli}

\section{(2) OpenEdition \\ 1 Journals}

\section{Edizione digitale}

URL: https://journals.openedition.org/studifrancesi/45855

DOI: $10.4000 /$ studifrancesi.45855

ISSN: 2421-5856

\section{Editore}

Rosenberg \& Sellier

\section{Edizione cartacea}

Data di pubblicazione: 1 octobre 2007

Paginazione: 444-445

ISSN: 0039-2944

\section{Notizia bibliografica digitale}

Stefania Carli, «L'«Encyclopédie méthodique» (1782-1832). Des Lumières au Positivisme, textes publiés par Claude Blanckaert et Michel Porret», Studi Francesi [Online], 152 (LI | II) | 2007, online dal 30 novembre 2015, consultato il 24 novembre 2021. URL: http://journals.openedition.org/studifrancesi/ 45855 ; DOI: https://doi.org/10.4000/studifrancesi.45855

Questo documento è stato generato automaticamente il 24 novembre 2021.

\section{cc) (†) $\odot$}

Studi Francesi è distribuita con Licenza Creative Commons Attribuzione - Non commerciale - Non opere derivate 4.0 Internazionale. 


\title{
L' «Encyclopédie méthodique» (1782-1832). Des Lumières au Positivisme, textes publiés par Claude Blanckaert et Michel Porret
}

\author{
Stefania Carli
}

\section{NOTIZIA}

L'«Encyclopédie méthodique» (1782-1832). Des Lumières au Positivisme, textes publiés par Claude BLANCKAERT et Michel PORRET, avec la collaboration de Fabrice BRANDLI, Genève, Librairie Droz, 2006 («Bibliothèque des Lumières», vol. LXVIII), pp. 830.

1 Il volume raccoglie ventotto studi sull'Encyclopédie méthodique, un'opera colossale costituita da più di duecento tomi apparsi tra il 1782 e il 1832. Ideata da Charles-Joseph Panckoucke e da lui pubblicata insieme al genero Henri Agasse e alla figlia Pauline, l'Encyclopédie méthodique è assai meno conosciuta dell' Encyclopédie di Diderot e d'Alembert, ma proprio per questo meritevole di essere riscoperta. Composta da ben cinquanta dictionnaires, essa viene presentata da Michel PORRET (Savoir encyclopédique, encyclopédie des savoirs, pp. 13-53) come un'opera che, durante la sua redazione, ha dovuto fare i conti con le numerose difficoltà causate dai cambiamenti politici e culturali intervenuti in Francia tra fine Settecento e inizio Ottocento, cioè nel periodo in cui la Méthodique fu composta e pubblicata.

Il grosso volume è suddiviso in quattro sezioni. Nella prima, dedicata alle «Sciences de l'homme», Claude BLANCKAERT (Le «circuit» de l'anthropologie. Figures de l'homme naturel et social dans le système méthodique des savoirs, pp. 69-102) insiste sul ruolo dell'antropologia, mentre Fernando VIDAL (Logique, métaphysique, morale, éducation: réformer les sciences humaines pour accomplir les Lumières, pp. 103-126) presenta il Dictionnaire de Logique, Métaphysique et Morale, apparso tra il 1786 e il 1791 e ispirato alle 
teorie di Locke e di Lacretelle. Questa sezione prende anche in considerazione il Dictionnaire d'Histoire, per la stesura del quale Nathalie RICHARD (Histoire, morale et politique à la fin du XVIII siècle: le «Dictionnaire d'Histoire» de l'«Encyclopédie méthodique», pp. 127-152) si chiede quanto possano aver inciso i fatti rivoluzionari del 1789, e quello di Théologie, redatto dall'anti-philosophe Bergier e qui analizzato da Didier MASSEAU (Un apologiste au service de l'«Encyclopédie méthodique»: Bergier et le dictionnaire de "Théologie", pp. 153-168). In questa prima parte sono studiati anche i Dictionnaires relativi all'Antichità (Anne-Marie MERCIER-FAIVRE, Les Antiquités dans l'«Encyclopédie méthodique»: un rendez-vous manqué avec le primitivisme, pp. 169-184) e alla geografia (Isabelle LABOULAIS-LESAGE, Les géographies de l'«Encyclopédie méthodique», pp. 185- 211).

3 La seconda sezione riguarda le «Sciences sociales, politiques ou économiques», discipline sulle quali gli avvenimenti storici, culturali e politici del tempo hanno influito in modo tutto particolare. Come ricorda Catherine LARRÈRE ( $L$ '«Encyclopédie méthodique»: une économie très politique, pp. 215-240), le innovative idee di Adam Smith hanno permesso evidenti progressi nel campo dell'economia politica, così come la nascita del nuovo governo americano ha indubbiamente interessato il diritto francese dell'epoca (Roberto MARTUCCI, Les articles «américains» de Jean-Nicolas Desmeunier et le droit public moderne, pp. 241-264). Per quanto riguarda l'economia politica, Fabrice BRANDLI (Entre utopie et réforme, les «projets chimériques» dans l'«Economie politique et diplomatique», pp. 265-310) non trascura il ruolo dell'utopia nella Méthodique, ma di notevole interesse è soprattutto lo studio della giurisprudenza, una delle materie che, a causa dei cambiamenti attuati dalla Rivoluzione francese, ha creato maggiori problemi ai redattori ingaggiati da Panckoucke (Fançoise BRIEGEL, «Asservir les jugements à la lettre, c'est en hannir la justice»: la "jurisprudence" de l'«Encyclopédie méthodique», pp. 311-340 e Fanny SIAM, Le dictionnaire de "Police et Municipalités» 1789-1791: la philosophie politicojuridique de Jacques Peuchet au service de l'administration, pp. 341-360). Questa seconda sezione si chiude con l'analisi dei Dictionnaires dedicati all'arte militare (Marc BELISSA, L'“Art militaire" de l'«Encyclopédie méthodique», pp. 361-380 e Marco CICCHINI, La médecine militaire: science médicale ou art de la guerre? Le mot et la chose dans l'«Encyclopédie méthodique», pp. 381-406) e allo studio dell'impatto avuto dagli eventi rivoluzionari sulla politica e l'economia del paese (Edna Hindie LEMAY, "Assemblée nationale constituante”: le passé est-il suspect face à la Révolution française?, pp. 407-420).

La terza parte del volume, incentrata sulle «Sciences naturelles, sciences exactes, techniques», è probabilmente la più rappresentativa. Vi sono studiati i Dictionnaires che si occupano di matematica (Jean DHOMBRES, L'aventure épistémologique de la mathématisation des Lumières éclairée par les images de marine de l'«Encyclopédie méthodique», pp. 423-466) e quelli dedicati ad alcune delle scienze più in evoluzione, ovvero la fisica (Hugues СНАВот, La «Physique» dans l'«Encyclopédie méthodique», entre sciences naturelles et sciences mathématiques, pp. 467-492) e la chimica (Patrice BRET, Les chimies de l'«Encyclopédie méthodique»: une discipline académique en révolution et des traditions d'atelier, pp. 521-552). Se la matematica trattata nella Méthodique non si allontana di molto da quella che era stata presentata nell'Encyclopédie di Diderot e d'Alembert (Alain COSTE e Pierre CRÉPEL, Prospectus pour une étude du dictionnaire des "Mathématiques" de l'«Encyclopédie méthodique», pp. 493-520), altre scienze evidenziano, oltre al legame con il passato, anche l'interesse per le nuove spinte innovative: è il caso del Dictionnaire de Botanique, iniziato prima della Rivoluzione e ultimato solo durante la Restaurazione (Denis LAMY, Les cryptogames dans la "Botanique" de l'«Encyclopédie 
méthodique», pp. 553-578), e degli studi sulla zoologia, ispirati sia alle teorie di Buffon sia a quelle, più recenti, di Linneo (Pascal DURIS, Entre Buffon et Linné: la zoologie dans l'«Encyclopédie méthodique», pp. 579-604). Chiude questa sezione l'approfondimento di Gilles DENIS sull'agricoltura (L'Agriculture" dans l'«Encyclopédie méthodique»: débauché d'une nouvelle discipline?, pp. 605-624), una disciplina che conosce la sua autonomia proprio grazie all'Encyclopédie di Panckoucke.

5 L'ultima sezione «Beaux-arts et musique, vocabulaire esthétique et travail de la langue», racchiude alcuni di quei settori disciplinari che più erano stati trascurati nella precedente e più nota opera di Diderot e d'Alembert; vi spiccano gli studi sulle Belle arti (Martial GUÉDRON, Disputer et définir: Le dictionnaire des "Beaux-Arts" de la «Méthodique», pp. 627-650 e Bruno RACAlButo, Ambitions et sources du dictionnaire des "Beaux-Arts", pp. 651-690). Il Dictionnaire d'architecture, a causa della continua evoluzione dei canoni estetici, si presenta invece come una sorta di fallimento (Laurent BARIDON, Le dictionnaire d'“Architecture" de Quatremère de Quincy: codifier le néoclassicisme, pp. 691-718). Concludono quest'ultima sezione e il volume gli studi dedicati al Dictionnaire de Musique (Alain CERNUSCHI, Les volumes "Musique" de l'«Encyclopédie méthodique». Stratification du texte, articulation du domaine, pp. 719-730), al Dictionnaire des Ana (Béatrice BRAUD, Le «Dictionnaire des Ana» ou les bons mots encyclopédiques, pp. 731-756) e al Vocabulaire universel di Panckoucke, che avrebbe dovuto chiudere l'opera ma che non venne mai pubblicato (Martine Groult, Les Vocabulaires de Panckoucke, pp. 757-762).

6 Lavoro colossale e di difficile attuazione, l'Encyclopédie méthodique è stata segnata dai cambiamenti politici e dalle trasformazioni scientifiche di un'epoca particolarmente turbolenta (Bronislaw BACZKo, Les trois temps de la "Méthodique", pp. 763-797), ma proprio per questo essa è un eccellente testimone dell'evoluzione culturale francese tra la fine dell'Illuminismo e l'inizio del Positivismo. 